\title{
3D Microanalysis of Biological Specimen Using FIB SEM
}

\author{
K. Scott
}

National Institute of Standards and Technology, 100 Bureau Drive MS-8372, Gaithersburg, MD 20899

Biological specimens are inherently 3-dimensional (3D) objects and the investigation of these complex and interconnected structures can greatly benefit from 3D imaging at varying resolution ranges. And, when combined with the distribution patterns of chemical species or elements in the cellular environment, 3D structural information can provide important insights into cellular processes and behavior.

Traditionally, much of the lower resolution imaging of tissues and cells has been accomplished by light microscopy while the high resolution ultra-structural imaging has been carried out using scanning electron microscopy (SEM) or transmission electron microscopy (TEM). However, 3D imaging of biological specimens has been largely limited to confocal light microscopy although TEM tomographic techniques and single particle techniques are sometimes used for 3D analysis of thin sectioned (50 nm - $100 \mathrm{~nm}$ thick sections) or small particulate (e.g. viruses) samples. Recent advances in light microscopy techniques have enabled 3D imaging of cells at resolutions well below diffraction limits [1]. However, many of these super resolution techniques are based on fluorescence microscopy and cannot achieve comparable resolution for general ultra-structural investigation.

Focused ion beam scanning electron microscopy (FIB SEM) has been used to produce high resolution 3D imaging of biological samples at $2 \mathrm{~nm}-5 \mathrm{~nm}$ lateral resolution and $20 \mathrm{~nm}-30 \mathrm{~nm}$ in axial resolution [2,3]. Additionally, FIB SEM combined with energy dispersive x-ray spectrometry (EDS) can provide 3D elemental information about the sample [4,5]. This ability to collect SEM resolution 3D structural data supplemented by chemical information can greatly enhance our understanding of biological processes. However, 3D microanalysis of biological specimens using FIB SEM faces several major challenges such as beam sensitivity of biological materials and low signal to noise ratio problem that often accompanies low voltage microanalysis.

We performed 3D microanalysis of diatoms in order to demonstrate and highlight the challenges associated with the use of FIB SEM based 3D elemental analysis for biological specimens. Both types of EDS detectors - $\mathrm{Si}(\mathrm{Li})$ and silicon drift - were used for the 3D microanalysis of diatoms. We found that the axial resolution of the technique depends mainly on the beam energy because higher beam energy translates into larger beam sample interaction depth as well as damage layer depth. The main challenge was in optimizing the experimental conditions to simultaneously maximize detection efficiency and minimize sample damage. Figure 1 shows a typical FIB SEM experimental geometry. Figure 2 illustrates the severity of sample damage even at relatively low voltage analysis conditions. The SEM images of the sample at several different axial/depth locations (surface, $300 \mathrm{~nm}, 500 \mathrm{~nm}$, and $800 \mathrm{~nm}$ below the surface) were collected after a 15 minute (clock time) mapping at $5 \mathrm{keV}$ beam energy and $0.17 \mathrm{nA}$ beam current. Despite the low beam energy, the damage layer extended past $500 \mathrm{~nm}$ into the sample surface and severe enough to obscure structural information. 
References

[1] J.W. Shaevitz, Nat. Methods 5 (2008) 471-472.

[2] J.A.W. Heymann et al., J. Struct. Biol. 155 (2006) 63-73.

[3] L.A. Giannuzzi et al., J. Oral. Maxillofac. Surg. 65 (2007) 737-747.

[4] P.G. Kotula et al., Microsc. Microanal. 12 (2006) 36-48.

[5] M. Schaffer et al., J. Ultramic. 107(2007) 587-597.

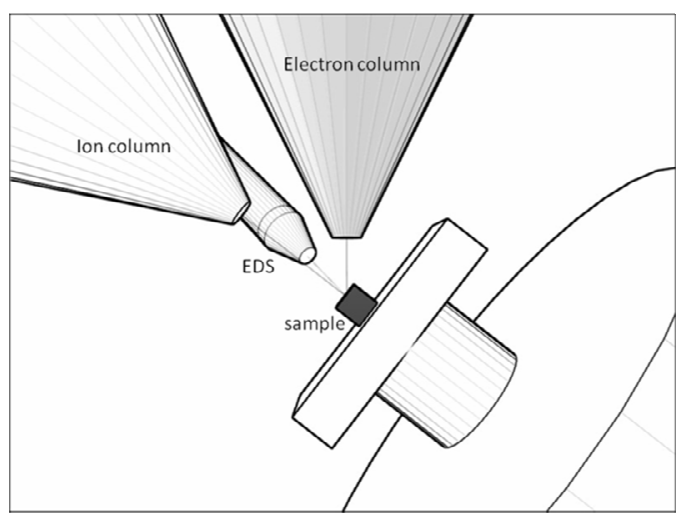

FIG. 1. Experimental geometry of the FIB SEM chamber showing the respective locations of electron column, ion column, EDS detector and sample.

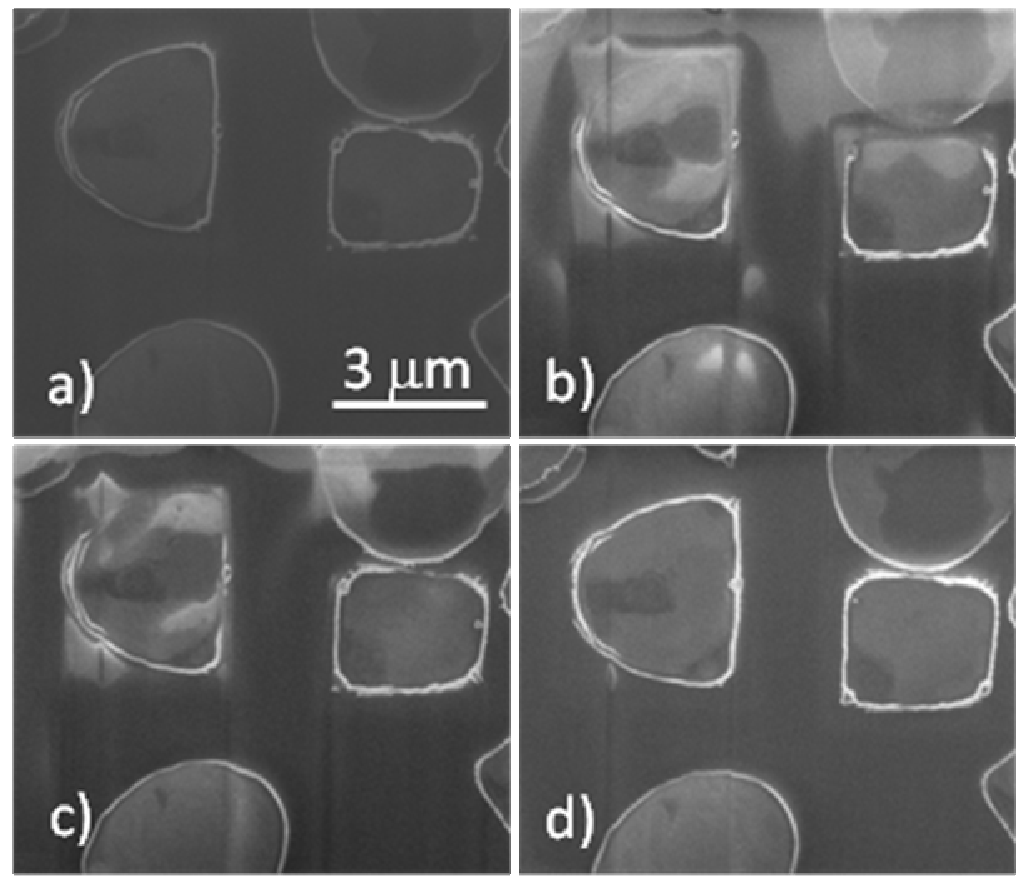

FIG. 2. Sample damages from $5 \mathrm{kV}$ mapping are noticeable at locations as deep as $500 \mathrm{~nm}$ into the sample. SEM images of a) the sample face where X-ray map was collected, b) $300 \mathrm{~nm}$, c) $500 \mathrm{~nm}$ and d) $800 \mathrm{~nm}$ below the analysis face. 